\title{
THE RHIC IONIZATION BEAM PROFILE MONITOR
}

\author{
P. Cameron, R. Connolly, R. Michnoff, V. Radeka, W. Ryan, T. Shea, \\ R. Sikora, D. Stephani, S. Tepikian, and N. Tsoupas \\ Brookhaven National Lab, Upton, NY \\ L. Woodworth \\ Raytheon Electronic Systems Div., Sudbury, MA
}

\begin{abstract}
Four ionization beam profile monitors (IPM's) have been installed in RHIC to measure vertical and horizontal profiles in the two rings. Each IPM collects and measures the distribution of electrons in the beamline resulting from residual gas ionization during bunch passage. The electrons are swept transversely from the beamline and collected on strip anodes oriented parallel to the beam axis. At each bunch passage the charge pulses are amplified, integrated, and digitized. The system is designed to measure single-bunch profiles with up to 120 bunches in each ring.
\end{abstract}

\section{INTRODUCTION}

The Relativistic Heavy Ion Collider (RHIC) at Brookhaven National Lab will accelerate and store beams of ions ranging from protons to gold nuclei [1]. Transverse beam profiles will be obtained by measuring the distribution of free electrons formed by beam ionization of the residual gas [2,3]. The electrons are swept from the beamline by a transverse electric field, amplified by a microchannel plate (MCP), and collected on a circuit board with strip anodes oriented parallel to the beam axis. A uniform magnetic field, parallel to the sweep electric field, counters the defocusing effects of space charge and recoil momentum.

The RHIC IPM detector is a close copy of the prototype, ref. 2, which was tested in the AGS-RHIC transfer line in 1997. However the prototype measured bunches separated by several seconds so it was adequate to use slow charge-sensitive amplifiers $(\tau \sim 4 \mu \mathrm{s})$, track-andhold amplifiers, and slow digitizers. The RHIC monitors are intended to digitize individual bunches spaced $107 \mathrm{~ns}$ apart. This requires faster preamplifiers and digitizers. Also much of the ground plane on the collector board is removed to lower capacitance. Finally a test pulse input was added to the detector to make it possible to determine gain and offset corrections for all 64 channels in a single measurement.

This paper describes the detector, digitizers and datacollection systems. The kinematics of electron confinement by the magnet field is discussed in ref. 2 .

\section{DESIGN CONSIDERATIONS}

RHIC will accelerate and store many different beams and the IPM's are located $50 \mathrm{~m}$ from an interaction point where the lattice optics varies with the requirements of the experiment. Depending on energy, ion species and i.p. optics the rms width of the beam can vary from $0.7 \mathrm{~mm}$ to $6.5 \mathrm{~mm}$. The channel spacing has to be small enough that a reasonable measurement can be made on the most narrow beam but the collector has to be wide enough to see the largest beam with some allowance for missteering and betatron oscillations.

The compromise chosen was to build a collector with 64 channels spaced $0.6 \mathrm{~mm}$ apart. Only $6 \sigma$ of the widest beam fits on the collector and $6 \sigma$ of the narrowest beam fits on only 7 channels. However the measurement of a Gaussian beam of $\sigma=0.7 \mathrm{~mm}$ with a collector $0.6 \mathrm{~mm}$ wide yields a rms width less than $4 \%$ larger than the true width.

RHIC has superconducting magnets so the residual gases are helium and hydrogen. The measured primary ionization rate for minimally ionizing protons in these two gases at atmospheric pressure is about 5.5 ion pairs/cm and the cross section is proportional to $\mathrm{Z}^{2}$ [4]. A gold bunch with $10^{9}$ ions in a vacuum of $10^{-8}$ torr will create about 4,000 ion/electron pairs in one passage. An $\mathrm{MCP}$ has a detection efficiency for $1.5 \mathrm{kV}$ electrons of $36 \%$ so about 1,400 electrons will be detected per bunch [5]. Monte Carlo simulations show that the variance of a Gaussian parent distribution can be obtained to within $\pm 2.2 \%$ by sampling 1000 points.

A bunch of $10^{11}$ protons will produce only $\sim 20$ detected electrons. In the beginning proton-beam profiles will be generated by summing up 50 or more single bunch signals. This can either be done with all bunches or by looking at the same bunch on consecutive turns. Single proton bunches can only be measured if a pressure bunch is added.

\section{DETECTOR}

Figure 1 is a cross section of the IPM from the perspective of looking along the beamline. The detector vacuum chamber is made from $10 \times 15 \mathrm{~cm}$ rectangular 304 stainless steel tubing. A detector port is formed by welding a short section of this tubing into the side with a 
10" conflat flange attached. The detector (fig. 2) is mounted entirely on a mating flange.

Two 30-cm long ' $\mathrm{C}$ ' permanent dipole magnets producing $0.14 \mathrm{~T}$ are placed $30 \mathrm{~cm}$ apart along the chamber. One is centered on the detector to focus the electrons and the second corrects the beam deflection caused by the first. The magnet pole tips are shaped to produce parallel field lines within an area of $38 \mathrm{~mm}$ parallel to the collector by $63 \mathrm{~mm}$ perpendicular to it. Within this collection area the maximum field-line transverse runout is $0.034 \mathrm{~mm}$ or $6 \%$ of a channel width.

The collector board is alumina metallized with gold. There are 64 anodes which are $91 \mathrm{~mm}$ long and spaced $0.6 \mathrm{~mm}$ apart. Plated-through holes connect the anodes to traces on the back of the board which bring the collected charge to the edge. Short alumina coated wires are soldered to pads on the board and are attached to two 35pin feedthroughs with push-on connectors. A chevron $8 \times 10 \mathrm{~cm}$ microchannel plate detector [6] is screwed directly to the board with a kapton gasket providing electrical insulation. The transverse sweep field is generated with an electrode and secondary-electron suppression grid on the other side of the beam.

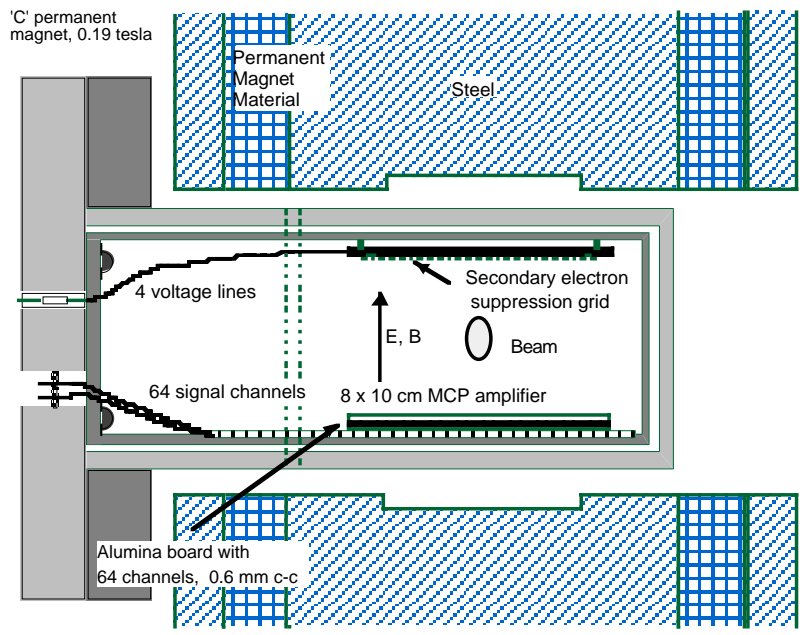

Figure 1. Cross sectional view of IPM. View is looking along the beamline. In this orientation the IPM would measure horizontal profiles.

Taut-wire beam-coupling measurements showed that the collector board needed to be shielded from the beam and that the MCP electrodes did not provide good rf shielding. The board is enclosed in a phosphor bronze shield and the input of the channel plate is covered with copper mesh with $\sim 90 \%$ OAR. Capacitors soldered around the edge of the mesh provide bypassing and the structure is grounded to the vacuum chamber with rf finger stock.

Four high-voltage lines bias the MCP input and output and the sweep electrode and suppression grid. The sweep voltage power supply floats on the MCP input voltage so the sweep field remains a constant $3.0 \mathrm{kV}$ as the MCP bias is adjusted. The MCP bias voltage is the only gain control ' $k$ nob' in the system. MCP bias is adjusted until the channel with the largest signal approach the dynamic ceiling of the electronics.

The detector flange has four 2.75 " conflat ports. Two are for signal feedthroughs and one is for the high voltage feedthrough. The top port in fig. 2 has a quartz window for illuminating the MCP with UV light. Although the illumination across the plate is not uniform, it will provide a method to baseline the new MCP. The current illumination pattern can be compared to the baseline to tell when the plate ages and the gain drops in the center channels.

Each collector anode capacitively couples to the output electrode of the MCP by about $1.3 \mathrm{pF}$. A step pulse delivered to the MCP output drives all 64 channels with pulses that only vary by the small differences in anode areas, differences which also affect the beam signals. The bias line to the MCP output is a parallel transmission line with $\mathrm{Z} \approx 105 \Omega$. This pulse input will be used to calibrate each detector to compensate in software for channel-to-channel variations in gain and offset.

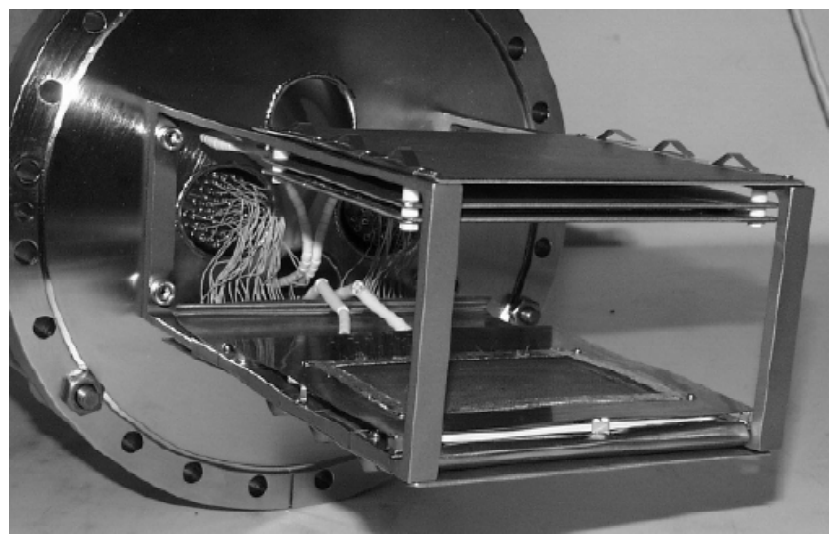

Figure 2. Photograph of detector. The MCP and anode board are on the bottom.

\section{ELECTRONICS}

Each bunch passage will deliver charge pulses to the anodes. Individual pulses are integrated and shaped by charge-sensitive, hybrid preamplifiers and hybrid shapers both designed by BNL Instrumentation Division. The preamplifier has a charge sensitivity of $0.6 \mathrm{~V} / \mathrm{pC}$ and a decay time constant of $20 \mathrm{~ns}$, and the shaper has a voltage gain of 10. Bench tests done by injecting signal directly into the prototype IPM measured a noise floor for the system of 2-3fC. The shaper output is amplified by a AD811 op amp [7] set to a gain of 2, and coupled into a $100 \Omega$ balanced line via a 1:1 pulse transformer.

Every channel also has a low-pass-filtered output which rolls off at about $100 \mathrm{kHz}$. These channels provide dc coupling to measure beam profiles with unbunched 
beams. After filtering the signals are amplified by OP-37 op amps and coupled into twisted-pair cables through SSM2142 balanced-line drivers [7].

Eight preamp circuits are on a $15 \times 15 \mathrm{~cm}$ board. Four of these boards are mounted in a chassis box which is attached to the vacuum feedthrough to minimize signal lead length. The pulse outputs are connected to VME digitizers installed in racks beneath the beamline to minimize cable lengths. The filtered signals are brought out of the tunnel to the instrumentation control room (ICR) on twisted pair cable and digitized with a RHIC general purpose $\mathrm{A} / \mathrm{D}$ converter [8]. All eight pulse channels on each preamp board are summed together into one signal which is brought back to the ICR on coaxial cable for diagnostic purposes.

The fast pulses have a base width of 100ns and a frequency of $9.4 \mathrm{MHz}$ with 120 bunches in a ring. They are digitized by Hytec VTR2535 8-channel VME digitizers [9]. Each digitizer channel has a dedicated AD9220 12-bit, 10MSPS ADC [7] backed with 128k words of memory. The digitizers are triggered by a V124 trigger module synchronized to the beam-sync clock [10]. During beam measurement the data are written into memory on the digitizer cards. Acquisition is then stopped and the data are read by the front end computer in the VME chassis and sorted into profiles.

\section{CONTROL PROGRAM}

During the commissioning of RHIC the IPM will be an experimental device which is operated manually from the ICR. The power supplies for both the MCP bias and the sweep field will be controlled from their front panels although they will be interlocked to vacuum gauges. Early commissioning will be done with only a few bunches in the ring so neither gain depletion or saturation is a concern. Eventually the measurement application will control these voltages.

The control application allows the operator to select a bunch and to select the number of turns to be collected. When the selected number of profiles are acquired, the program sums the data into one average profile. It fits a Gaussian to the profile and displays both the measured profile and the center and variance of the fit. If desired this can be repeated at a selected interval. After each data set is collected the new profile and width are displayed. The last profile is added to a mountain-range display and the values of the width and center are added to a strip-chart display. In this manner we can follow the beam profile during the energy ramp.

\section{SUMMARY}

Four IPM's are installed in RHIC to measure horizontal and vertical beam profile in both rings. The design is based on a prototype which measured single-bunch profiles in the AGS-RHIC transfer line. We expect to be able to measure single-bunch profiles of gold beams and averaged profiles of proton beams. Single-bunch profiles of proton beams will only be possible with a pressure bump.

\section{ACKNOWLEDGMENTS}

We thank Phil Cerniglia for supervising the cable installation and John Cupola for assembling much of the hardware. Doug Hunter and Pedro Solano assembled the magnets and John Daddario terminated most of the cables. Also thanks to Animesh Jain for mapping the magnet and Chau Lac for designing the magnet stands. Jim Zagel alerted us to the problem of rf coupling through the MCP. This work was done under the auspices of the U.S. Department of Energy.

\section{REFERENCES}

1. http://www.rhichome.bnl.gov/RHIC/index.html

2. R. Connolly, P. Cameron, W. Ryan, T.J. Shea, R. Sikora, and N. Tsoupas, "A Prototype Ionization Profile Monitor for RHIC," Proceedings of the 1997 Particle Accelerator Conference, Vancouver, BC.

3. W.H. DeLuca, "Beam Detection Using Residual Gas Ionization", IEEE Trans. Nucl. Sci., NS-16, 813 (1969).

4. F. Sauli, "Principles of Operation of Multiwire Proportional and Drift Chambers," CERN 77-09 (1977).

5. J. Wiza, "Microchannel Plate Detectors," Nucl. Instr. Meth. 162 (1979) 587-601.

6. Galileo Electro-Optics Corp., Sturbridge, MA 01566.

7. Analog Devices, Norwood, MA 02062-9106.

8. Michnoff, R. "The RHIC General Purpose Multiplexed Analog-to-Digital Converter System", Proceedings of the 1995 Particle Accelerator Conference, Dallas, TX.

9. Hytec Electronics Ltd., Reading, Berks. RG20JT, UK.

10. H. Hartmann and T. Kerner, "RHIC Beam Synchronous Trigger Module," these proceedings. 